\title{
Glutathione depletion modulates methanol, formaldehyde and formate toxicity in cultured rat conceptuses
}

\author{
C. Harris ${ }^{1}$, M. Dixon ${ }^{1}$ and J.M. Hansen ${ }^{2}$ \\ ${ }^{1}$ Toxicology Program, Department of Environmental Health Sciences, University of Michigan, Ann \\ Arbor, Michigan and ${ }^{2}$ Department of Biochemistry, Emory University, Atlanta, Georgia, USA
}

Received 20 November 2003; accepted 26 March 2004

Keywords: embryo, formaldehyde, formic acid, glutathione, methanol, visceral yolk sac

\begin{abstract}
The proposed use of methanol $\left(\mathrm{H}_{3} \mathrm{COH}\right)$ as an alternative to fossil fuels has prompted concern about potential health risks resulting from widespread environmental exposure. Methanol is teratogenic in rodents and, although the exact toxic species is not known, teratogenesis may result from the enzymatic biotransformation of $\mathrm{H}_{3} \mathrm{COH}$ to formaldehyde $\left(\mathrm{CH}_{2} \mathrm{O}\right)$ and formic acid causing increased biological reactivity and toxicity. A protective role for the antioxidant glutathione (GSH) has been described for $\mathrm{H}_{3} \mathrm{COH}, \mathrm{CH}_{2} \mathrm{O}$ and formic acid toxicity in various biological systems but has yet to be evaluated in the developing conceptus. Whole embryo culture studies were conducted using GD 10-11 rat conceptuses to elucidate the relationship between $\mathrm{H}_{3} \mathrm{COH}$ and its metabolites and GSH status. Methanol exposure produced a decrease in normal growth parameters and a dose-dependent loss of viability. $\mathrm{CH}_{2} \mathrm{O}$ had deleterious effects on embryo growth and viability. Sodium formate (HCOONa) exposure resulted in a high mortality rate but viable embryos did not manifest any abnormalities. Methanol, $\mathrm{CH}_{2} \mathrm{O}$, and $\mathrm{HCOONa}$ all produced a significant depletion of GSH in both embryo and VYS. Inhibition of GSH synthesis by L-buthionine- $S, R$ sulfoximine (BSO) treatment exacerbated $\mathrm{H}_{3} \mathrm{COH}, \mathrm{CH}_{2} \mathrm{O}$ and $\mathrm{HCOONa}$ embryotoxicity. Interestingly, only $\mathrm{H}_{3} \mathrm{COH} / \mathrm{BSO}$ and $\mathrm{CH}_{2} \mathrm{O} / \mathrm{BSO}$ co-treatments caused increased malformation, while embryos treated with $\mathrm{HCOONa} / \mathrm{BSO}$ did not produce any developmental deformities. These results implicate $\mathrm{CH}_{2} \mathrm{O}$ as the most embryotoxic $\mathrm{H}_{3} \mathrm{COH}$ metabolite, on a molar basis, in terms of causing dysmorphogenesis, alterations of normal growth parameters and embryolethality. HCOONa was selectively embryolethal and did not produce dysmorphogenesis. $\mathrm{CH}_{2} \mathrm{O}$ toxicity is potentiated by GSH depletion, indicating that GSH may be more directly involved in its detoxication in the embryo.
\end{abstract}

Abbreviations: ADH1, alcohol dehydrogenase 1; ADH3, alcohol dehydrogenase 3; BSO, Lbuthionine- $S$, $R$-sulfoximine; $\mathrm{CH}_{2} \mathrm{O}$, formaldehyde; DPTA, diethylenetriaminepentaacetic acid; FA, formic acid; GSH, glutathione; $\mathrm{H}_{3} \mathrm{COH}$, methanol; HBSS, Hank's balanced salt solution; HEPES, N-[2-hydroxyethyl]-piperazine-N'-[3-propane-sulfonic acid]; MSA, methanesulfonic acid; HCOONa, sodium formate; ROS, reactive oxygen species; VYS, visceral yolk sac 


\section{Introduction}

Methanol $\left(\mathrm{H}_{3} \mathrm{COH}\right)$ has a variety of uses, such as in agricultural, laboratory, industrial, and domestic settings primarily as solvent but is also present in cosmetic formulations $(0.1 \%$ to $5 \%$ of the preparation (Food and Drug Administration, 1984), foods such as fruits, juices $(140 \mathrm{mg} / \mathrm{L})$, and vegetables (Lanigan, 2001) and fermented beverages $(1.5 \mathrm{~g} / \mathrm{L}$ ) (Von Burg, 1994). Moreover, $\mathrm{H}_{3} \mathrm{COH}$ may become even more commonplace for human exposure as it has been suggested as a fossil fuel alternative. As $\mathrm{H}_{3} \mathrm{COH}$ becomes more frequently used by the public, understanding $\mathrm{H}_{3} \mathrm{COH}$ developmental toxicity will become an increasingly important issue.

Methanol has been described to have detrimental effects on development in several animal models following exposure by either inhalation or oral routes. Among rodents, both rat and mouse embryos have been shown to be directly sensitive to $\mathrm{H}_{3} \mathrm{COH}$ in whole embryo culture, although, mice are considerably more sensitive (Andrews et al., 1995). Inhalation exposure of $\mathrm{H}_{3} \mathrm{COH}$ (>20000 ppm) to pregnant rats resulted numerous defects, including exencephaly, encephalocele and skeletal and visceral abnormalities (Nelson et al., 1985). Infurna and Weiss (1986) showed that methanol exposure via oral gavage causes similar types of effects in rats as were observed in inhalation studies, albeit not as severe, but also produced additional behavior abnormalities, such as the failure to suckle and home correctly. Even more serious developmental defects, such as cleft palate and exencephaly, were observed in CD-1 mice dosed orally with $\sim 4 \mathrm{mg} / \mathrm{kg} \mathrm{H}{ }_{3} \mathrm{COH}$ during organogenesis, from GD 6-15 (Bolon et al., 1993). In rats, a single oral dosing of $\mathrm{H}_{3} \mathrm{COH}$ resulted in the failure of testicles to descend properly, exopthalmia and anopthalmia, but did not produce any clinical manifestations in the dam (Youssef et al., 1997).
The toxicokinetics of $\mathrm{H}_{3} \mathrm{COH}$ implicate the developing fetus as a sensitive target for $\mathrm{H}_{3} \mathrm{COH}$-induced toxicity. Ward and Pollack (1996) showed that pregnant rats and mice did not eliminate $\mathrm{H}_{3} \mathrm{COH}$ as well as non-pregnant animals following a single bolus injection, only achieving approximately $60 \%$ of the non-pregnant dam elimination. In another study by the same group, pregnant dams were dosed with $\mathrm{H}_{3} \mathrm{COH}$ via bolus injection. Embryos had approximately $25 \%$ greater $\mathrm{H}_{3} \mathrm{COH}$ concentrations at distribution equilibrium than maternal tissues (Ward and Pollack, 1995). Due to excessive accumulation of $\mathrm{H}_{3} \mathrm{COH}$ during pregnancy and preferential $\mathrm{H}_{3} \mathrm{COH}$ buildup in the embryo proper, insight into mechanisms for $\mathrm{H}_{3} \mathrm{COH}$ metabolism and elimination is paramount for providing protection against $\mathrm{H}_{3} \mathrm{COH}$-induced developmental toxicity.

In adults, the majority of $\mathrm{H}_{3} \mathrm{COH}$ metabolism occurs in the liver, but an understanding how the conceptus metabolizes and detoxicates $\mathrm{H}_{3} \mathrm{COH}$ is lacking. Generally, $\mathrm{H}_{3} \mathrm{COH}$ is metabolized into formaldehyde $\left(\mathrm{CH}_{2} \mathrm{O}\right)$ via alcohol dehydrogenase 1 (ADH1), the predominate enzyme in humans and non-human primates involved in the initial steps of $\mathrm{H}_{3} \mathrm{COH}$ detoxication (Figure 1). It is also known that $\mathrm{CH}_{2} \mathrm{O}$ can be formed from $\mathrm{H}_{3} \mathrm{COH}$ through a hydroxyl radical-mediated pathway. Formaldehyde does not accumulate in tissues as it is rapidly converted by its reaction with glutathione (GSH) to form $S$ formylglutathione, which is mediated by the NAD-dependent enzyme, formaldehyde dehydrogenase or alcohol dehydrogenase 3 (ADH3). Thiolase converts $S$-formylglutathione to formic acid (FA), which is widely believed to be the species that results in various birth defects. The third and terminal reaction that converts formate to $\mathrm{CO}_{2}$ and water is catalyzed primarily by catalase (Kavet and Nauss, 1990; Von Burg, 1994).

Methanol exposure in rats via intraperitoneal injection caused a significant GSH deple- 


\section{METHANOL}

ADH1 1
CATALASE

FORMALDEHYDE

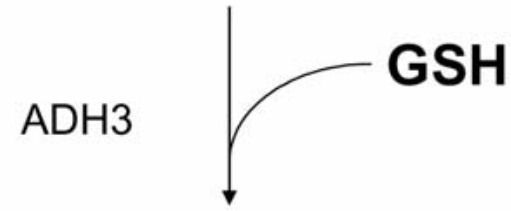

\section{S-FORMYLGLUTATHIONE}

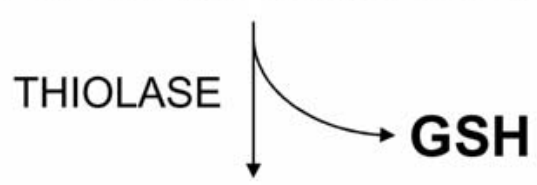

FORMIC ACID

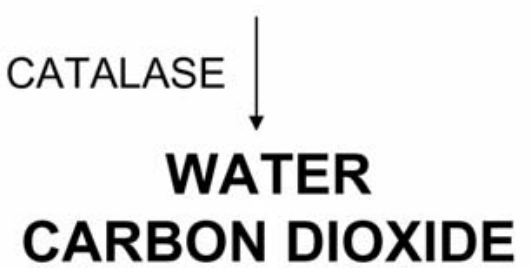

Figure 1. Methanol metabolism. Overview of the metabolism and the enzymes proposed to be involved in the conversion of methanol to water and $\mathrm{CO}_{2}$.

tion in the brain after 2 days $(\sim 23 \%)$ and 7 days $(\sim 52 \%)$ (Farbiszewski et al., 2000). In the same study, lipid peroxidiation was determined using the thiobarbituric acid-reacting substances (TBARS) assay. The rat brain showed increased levels of lipid peroxidation after 2 days (increased by $331 \%$ of control) and 7 days (increased by $340 \%$ of control) with $\mathrm{H}_{3} \mathrm{COH}$ exposure (Farbiszewski et al., 2000). Supplementation with antioxidants curbed both GSH depletion and lipid peroxidation. These findings suggest that $\mathrm{H}_{3} \mathrm{COH}$ may, directly or indirectly, cause oxidative stress in the brain and may also be present in other tissues as well, and implies an even greater role for antioxidants, like GSH.

Although GSH is an integral part of the $\mathrm{H}_{3} \mathrm{COH}$ detoxication pathway, its role has not been well-defined following $\mathrm{H}_{3} \mathrm{COH}$ exposure in respect to the developing conceptus. In the present study, we utilized whole embryo culture to evaluate the effect of $\mathrm{H}_{3} \mathrm{COH}$ and its metabolites $\left(\mathrm{CH}_{2} \mathrm{O}\right.$ and FA) on gross developmental parameters under conditions where GSH is depleted. These experiments may provide a greater understanding of how GSH contributes to $\mathrm{H}_{3} \mathrm{COH}$ detoxication in the embryo and identify which chemical species may be most responsible for teratogenesis and embryolethality.

\section{Materials and methods}

\section{Chemicals}

Methanol, formaldehyde, sodium formate, acetonitrile, methanesulfonic acid (MSA), diethylenetriaminepentaacetic acid (DPTA), $\mathrm{N}$-[2-hydroxyethyl]-piperazine- $\mathrm{N}^{\prime}$-[3-propanesulfonic acid] (HEPES) and L-buthionine- $S, R$ sulfoximine (BSO) were obtained from Sigma Chemical Co. (St Louis, MO). Monobromobimane (thiolyte) was obtained from Calbiochem (La Jolla, CA). Hank's balanced salt solution (HBSS) was acquired from Life Sciences (Grand Island, NY). Sodium methaneulfonate was purchased from Aldrich Chemical (Milwaukee, WI). All other reagents were obtained from common commercial sources. 


\section{Animals}

Primagravida Sprague-Dawley rats were obtained from the Reproductive Sciences Program Small Animal Core, University of Michigan on GD 6-9. Day 0 was determined by a sperm-positive vaginal smear on the morning following copulation. Pregnant rats were maintained on a 12-h light/12-h dark cycle until explantation on GD 10. Food and water were given ad libitum.

\section{Whole embryo culture}

Rat whole embryo culture was performed as described by Fantel and colleagues (1989) and Harris and colleagues (1987). Each rat conceptus (8-10 somites) was prepared for culture on GD 10 and placed in bottles (1 conceptus/ $\mathrm{ml}$ media) with warm media consisting of $33 \%$ $(\mathrm{v} / \mathrm{v})$ heat-inactivated pregnant rat serum and $66 \%$ HBSS (pH 7.4). Bottles containing conceptuses and media were placed in a rotating drum (BTC Engineering, Cambridge, UK) under constant gassing conditions of $20 \% \mathrm{O}_{2} /$ $5 \% \mathrm{CO}_{2} / 75 \% \mathrm{~N}_{2}(\mathrm{v} / \mathrm{v} / \mathrm{v})$ and maintained at $37^{\circ} \mathrm{C}$ for a $20 \mathrm{~h}$ period. Atmospheric conditions were changed to $95 \% \mathrm{O}_{2} / 5 \% \mathrm{CO}_{2}$ (v/v) during the final $4 \mathrm{~h}$ on the following day (GD 11) to meet the metabolic requirements of the developing conceptus. Conceptuses were exposed to varying concentrations of $\mathrm{H}_{3} \mathrm{COH}$ (12 and $24 \mathrm{mg} / \mathrm{ml}), \mathrm{CH}_{2} \mathrm{O}(3$ and $5 \mu \mathrm{g} / \mathrm{ml})$ and HCOONa $(0.5$ and $2 \mathrm{mg} / \mathrm{ml})$. Dose ranges were initially chosen based on other studies in whole embryo culture that produced optimal dysmorphogenesis while retaining a reasonably high viability (Andrews et al., 1995). Since very few $\mathrm{H}_{3} \mathrm{COH}$ studies have centered on $\mathrm{CH}_{2} \mathrm{O}$, concentrations were determined by the generation of separate dose-response profiles (data not shown). In some cultures, exposure to $\mathrm{H}_{3} \mathrm{COH}, \mathrm{CH}_{2} \mathrm{O}$ or $\mathrm{HCOONa}$ was preceded by prior treatment with BSO for $4 \mathrm{~h}$. After termination of culture, growth developmental parameters were evaluated, including viability, embryonic rotation, neuropore closure, crownrump length and somite formation. In addition, the visceral yolk sac (VYS) and embryo from $\mathrm{H}_{3} \mathrm{COH}(24 \mathrm{mg} / \mathrm{ml}), \mathrm{CH}_{2} \mathrm{O}(3 \mu \mathrm{g} / \mathrm{ml})$ and $\mathrm{HCOONa}(2 \mathrm{mg} / \mathrm{ml})$ treatment groups (both with and without BSO pretreatments) were each placed in separate microcentrifuge tubes containing $200 \mu \mathrm{l}$ of $200 \mathrm{mmol} / \mathrm{L}$ MSA for GSH and cysteine. Protein analysis was performed by the method of Bradford (Bradford, 1976). Measurements of GSH in embryos treated with $3 \mu \mathrm{g} \mathrm{CH}_{2} \mathrm{O} / \mathrm{ml}$ were analyzed rather than $6 \mu \mathrm{g} / \mathrm{ml}$ due to the increased toxicity at the higher $\mathrm{CH}_{2} \mathrm{O}$ concentration. Samples were snap-frozen in liquid nitrogen and placed in a freezer at $-70^{\circ} \mathrm{C}$ until prepared for HPLC analysis.

\section{HPLC analysis}

GSH and cysteine concentrations were measured using the HPLC method as described by Fahey and Newton (1987) and as modified by Harris and colleagues (1991). Frozen tissue samples were thawed and homogenized by ultrasonic disruption. Two hundred microliters of $4 \mathrm{~mol} / \mathrm{L}$ sodium methanesulfonate to complete protein precipitation were added to each sample followed by centrifugation at 13900 $\mathrm{rpm}$ for $5 \mathrm{~min}$. The resulting supernatants were removed and separately placed into new microcentrifuge tubes containing $160 \mu \mathrm{l}$ of 5 $\mathrm{mmol} / \mathrm{L}$ DPTA in $1 \mathrm{~mol} / \mathrm{L}$ Hepes $(\mathrm{pH} 8.5)$. Sample protein content was determined by solubilization of the pellet in $0.25 \mathrm{~mol} / \mathrm{L}$ $\mathrm{NaOH}$ and was followed by protein assay as described by Bradford (1976). Derivatization of the supernatant was carried out by the addition of $20 \mu \mathrm{l}$ of $0.2 \mathrm{mmol} / \mathrm{L}$ monobromobimane in acetonitrile. Samples were allowed to incubate for $20 \mathrm{~min}$ in the dark at room temperature. Derivatization was completed by the addition of $380 \mu \mathrm{l}$ of $400 \mathrm{mmol} / \mathrm{L}$ MSA after which samples were snap-frozen in liquid nitrogen and stored at $-70^{\circ} \mathrm{C}$. 
HPLC analyses were carried out by using a Waters NovaPak C 4- $\mu$ m Radial-Pak cartridge fitted with a NovaPak Guard-Pak guard precolumn. Samples were eluted using an isocratic mobile phase consisting of $14.2 \%$ methanol (v/ v) and $2.5 \%$ glacial acetic acid (v/v) at a flow rate of $1.0 \mathrm{ml} / \mathrm{min}$. Following elution of both the cysteine and GSH peaks, the column was washed with $90 \%$ methanol $(\mathrm{v} / \mathrm{v})$ and $2.5 \%$ glacial acetic acid (v/v) for $15 \mathrm{~min}$. Detection and quantitation of GSH-bimane and cysteinebimane was accomplished using a Waters Model 470 scanning fluorescence detector $(\lambda$ excitation $360 \mathrm{~nm} ; \lambda$ emission $455 \mathrm{~nm}$ ) followed by analysis and quantitation by a Waters Model 746 data module. Authentic standards were prepared and used to identify each peak of interest. These same standards were also used to quantify each sample. This method is sensitive enough to accurately detect GSH and cysteine levels as low as $10 \mathrm{pmol} /$ injection.

\section{Statistics}

Statistical analyses were performed using SigmaStat 2.0 software (Jandel Scientific, San Rafael, CA). Viability, rotation and neuropore closure significant differences were determined by a z-test comparison of proportions of normal vs. abnormal parameters. Crown-rump length, somite number, GSH concentrations and cysteine concentrations significant differences were determined by one-way ANOVA followed by a Tukey's posthoc test. Significant differences were established where $p<0.05$.

\section{Results}

\section{Growth and developmental parameters}

Methanol exposure. The addition of $\mathrm{H}_{3} \mathrm{COH}$ into the culture medium resulted in significant effects on one or more of the growth parameters. The results are presented in Table 1. At $12 \mathrm{mg} / \mathrm{ml}$ and $24 \mathrm{mg} / \mathrm{ml} \mathrm{H}_{3} \mathrm{COH}$, embryo viability and neuropore closure were significantly decreased. Similar trends were represented in the crown-rump measurement, where overall length was significantly decreased in all $\mathrm{H}_{3} \mathrm{COH}$ groups. Somite number decreased with all $\mathrm{H}_{3} \mathrm{COH}$ treatments and followed in a dose-dependent manner. Morphological observations of the conceptus included embryonic bloody blisters in treated embryos.

Formaldehyde exposure. Formaldehyde treatment (3 and $6 \mu \mathrm{g} / \mathrm{ml}$ ) yielded various effects on embryo development, which are tabulated in Table 2. Treatment with 3 and $6 \mu \mathrm{g} \mathrm{CH}_{2} \mathrm{O} /$ $\mathrm{ml}$ caused a significant decrease in embryo viability and rotation. Neuropore closure, crown-rump length and somite number measurements were unaffected at $3 \mu \mathrm{g} / \mathrm{ml}$ but were significantly different at $6 \mu \mathrm{g} / \mathrm{ml}$. At the lower dose $(3 \mu \mathrm{g} / \mathrm{ml})$, some blister formation was observed. At a dose of $6 \mu \mathrm{g} / \mathrm{ml}$, a greater number of embryos showed evidence of dysmorhphogenesis, including blisters and more extensive regions of apparent necrosis, identified by markedly increased tissue opacity.

Formate exposure. Sodium formate had little effects on growth and developmental parameters in surviving embryos as shown in Table 3. Embryonic viability was greatly affected with HCOONa treatments, but at the lower dose $(0.5 \mathrm{mg} / \mathrm{ml})$, the surviving embryos showed no developmental or growth dysmorphogenesis. Furthermore, in the higher dose $(2 \mathrm{mg} / \mathrm{ml})$, the only parameter that showed a significant change was somite number. One embryo at $0.5 \mathrm{mg} / \mathrm{ml}$ had blisters, and one embryo at $2 \mathrm{mg} / \mathrm{ml}$ appeared necrotic.

$B S O$ co-treatments. Buthionine- $S, R$-sulfoximine treatment alone had no significant effects on most of the growth and developmental parameters. The only parameter affected was crown-rump length, where BSO treated embryos were slightly, but significantly, smaller (Tables 1-3). 
Table 1. Treatment of GD 10-11 rat conceptuses with methanol (12-24 mg/ml) with or without BSO for $24 \mathrm{~h}$ in vitro.

\begin{tabular}{|c|c|c|c|c|c|c|}
\hline Treatment & $\begin{array}{c}\% \\
\text { Viability }\end{array}$ & $\begin{array}{c}\% \\
\text { Rotation }\end{array}$ & $\begin{array}{c}\% \\
\text { Closure }\end{array}$ & $\begin{array}{l}\text { Length (mm) } \\
\text { (n) }\end{array}$ & $\begin{array}{l}\text { Somites } \\
\text { (n) }\end{array}$ & $\begin{array}{l}\text { Malformations } \\
\text { (n) }\end{array}$ \\
\hline Control & $\begin{array}{c}100 \\
(30 / 30)\end{array}$ & $\begin{array}{c}97 \\
(29 / 30)\end{array}$ & $\begin{array}{c}93 \\
(27 / 29)\end{array}$ & $\begin{array}{c}3.4 \pm 0.0 \\
(29)\end{array}$ & $\begin{array}{l}25.5 \pm 0.3 \\
(22)\end{array}$ & None \\
\hline $\begin{array}{l}\mathrm{H}_{3} \mathrm{COH} \\
(12 \mathrm{mg} / \mathrm{ml})\end{array}$ & $\begin{array}{c}77^{*} \\
(27 / 35)\end{array}$ & $\begin{array}{c}96 \\
(26 / 27)\end{array}$ & $\begin{array}{c}77 * \\
(20 / 26)\end{array}$ & $\begin{array}{c}3.1 \pm 0.0 * \\
(26)\end{array}$ & $\begin{array}{c}22.1 \pm 0.5^{*} \\
(27)\end{array}$ & Blisters (4) \\
\hline $\begin{array}{l}\mathrm{H}_{3} \mathrm{COH} \\
(24 \mathrm{mg} / \mathrm{ml})\end{array}$ & $\begin{array}{c}60 * \\
(12 / 20)\end{array}$ & $\begin{array}{c}100 \\
(12 / 12)\end{array}$ & $\begin{array}{c}50^{*} \\
(6 / 12)\end{array}$ & $\begin{array}{l}2.7 \pm 0.2^{*} \\
\quad(12)\end{array}$ & $\begin{array}{c}19.0 \pm 0.0^{*} \\
(12)\end{array}$ & $\begin{array}{c}\text { Blisters (3) } \\
\text { Necrotic (7) }\end{array}$ \\
\hline $\begin{array}{l}\text { BSO } \\
(2 \mathrm{mg} / \mathrm{ml})\end{array}$ & $\begin{array}{c}96 \\
(27 / 28)\end{array}$ & $\begin{array}{c}96 \\
(26 / 27)\end{array}$ & $\begin{array}{c}96 \\
(25 / 26)\end{array}$ & $\begin{array}{c}3.2 \pm 0.0^{*} \\
(27)\end{array}$ & $\begin{array}{l}24.8 \pm 0.3 \\
\quad(26)\end{array}$ & None \\
\hline $\begin{array}{l}\mathrm{H}_{3} \mathrm{COH} \\
(12 \mathrm{mg} / \mathrm{ml}) \\
\mathrm{BSO}(2 \mathrm{mg} / \mathrm{ml})\end{array}$ & $\begin{array}{c}81 \\
(13 / 16)\end{array}$ & $\begin{array}{c}54 * * \\
(7 / 13)\end{array}$ & $\begin{array}{c}54 \\
(7 / 13)\end{array}$ & $\begin{array}{c}2.4 \pm 0.1 * * \\
(7)\end{array}$ & $\begin{array}{c}19.0 \pm 0.1^{* *} \\
(13)\end{array}$ & None \\
\hline $\begin{array}{l}\mathrm{H}_{3} \mathrm{COH} \\
(24 \mathrm{mg} / \mathrm{ml}) \\
\mathrm{BSO}(2 \mathrm{mg} / \mathrm{ml})\end{array}$ & $\begin{array}{c}50 \\
(5 / 10)\end{array}$ & $\begin{array}{l}20 * * \\
(1 / 5)\end{array}$ & $\begin{array}{c}0 * * \\
(0 / 5)\end{array}$ & $\begin{array}{c}2.0 \pm 0.0^{* *} \\
(5)\end{array}$ & NA & Blisters (5) \\
\hline
\end{tabular}

*Significant difference from control $(p<0.05)$.

**Significant difference from $\mathrm{H}_{3} \mathrm{COH}$ only $(p<0.05)$.

Table 2. Treatment of GD 10-11 rat conceptuses with formaldehyde (3-6 $\mu \mathrm{g} / \mathrm{ml})$ with or without BSO for $24 \mathrm{~h}$ in vitro

\begin{tabular}{|c|c|c|c|c|c|c|}
\hline Treatment & $\begin{array}{c}\% \\
\text { Viability }\end{array}$ & $\begin{array}{c}\% \\
\text { Rotation }\end{array}$ & $\begin{array}{c}\% \\
\text { Closure }\end{array}$ & $\begin{array}{l}\text { Length (mm) } \\
\text { (n) }\end{array}$ & $\begin{array}{l}\text { Somites } \\
\text { (n) }\end{array}$ & $\begin{array}{c}\text { Malformations } \\
\text { (n) }\end{array}$ \\
\hline Control & $\begin{array}{c}100 \\
(26 / 26)\end{array}$ & $\begin{array}{c}96 \\
(25 / 26)\end{array}$ & $\begin{array}{c}96 \\
(24 / 25)\end{array}$ & $\begin{array}{l}3.4 \pm 0.1 \\
(25)\end{array}$ & $\begin{array}{l}25.8 \pm 0.3 \\
\quad(27)\end{array}$ & None \\
\hline $\begin{array}{l}\mathrm{CH}_{2} \mathrm{O} \\
(3 \mu \mathrm{g} / \mathrm{ml})\end{array}$ & $\begin{array}{c}84^{*} \\
(21 / 25)\end{array}$ & $\begin{array}{c}81^{*} \\
(17 / 21)\end{array}$ & $\begin{array}{c}96 \\
(19 / 21)\end{array}$ & $\begin{array}{l}2.9 \pm 0.1 \\
(20)\end{array}$ & $\begin{array}{l}24.7 \pm 0.2 \\
\quad(18)\end{array}$ & Blisters \\
\hline $\begin{array}{l}\mathrm{CH}_{2} \mathrm{O} \\
(6 \mu \mathrm{g} / \mathrm{ml})\end{array}$ & $\begin{array}{c}60^{*} \\
(12 / 20)\end{array}$ & $\begin{array}{c}50 * \\
(6 / 12)\end{array}$ & $\begin{array}{c}42^{*} \\
(5 / 12)\end{array}$ & $\begin{array}{l}2.7 \pm 0.1 * \\
\quad(12)\end{array}$ & $\begin{array}{c}20 \pm 1.1 \\
(12)\end{array}$ & Blisters (10) \\
\hline $\begin{array}{l}\text { BSO } \\
(2 \mathrm{mg} / \mathrm{ml})\end{array}$ & $\begin{array}{c}92 \\
(24 / 26)\end{array}$ & $\begin{array}{c}95 \\
(23 / 24)\end{array}$ & $\begin{array}{c}96 \\
(22 / 23)\end{array}$ & $\begin{array}{c}3.2 \pm 0.0 * \\
(24)\end{array}$ & $\begin{array}{l}24.9 \pm 0.3 \\
\quad(21)\end{array}$ & None \\
\hline $\begin{array}{l}\mathrm{CH}_{2} \mathrm{O} \\
(3 \mu \mathrm{g} / \mathrm{ml}) \\
\mathrm{BSO}(2 \mathrm{mg} / \mathrm{ml})\end{array}$ & $\begin{array}{c}40 * * \\
(10 / 25)\end{array}$ & $\begin{array}{c}20 * * \\
(2 / 10)\end{array}$ & $\begin{array}{c}20 * * \\
(2 / 10)\end{array}$ & $2.2 \pm 0.1$ & $\begin{array}{c}22.7 \pm 0.8^{* *} \\
(10)\end{array}$ & $\begin{array}{l}\text { Blisters (3) } \\
\text { Necrotic (6) }\end{array}$ \\
\hline $\begin{array}{l}\mathrm{CH}_{2} \mathrm{O} \\
(6 \mu \mathrm{g} / \mathrm{ml}) \\
\mathrm{BSO}(2 \mathrm{mg} / \mathrm{ml})\end{array}$ & $\begin{array}{c}0^{* *} \\
(0 / 18)\end{array}$ & NA & NA & NA & NA & NA \\
\hline
\end{tabular}

Significant difference from control $(p<0.05)$.

$* *$ Significant difference from $\mathrm{CH}_{2} \mathrm{O}$ only $(p<0.05)$. 
Table 3. Treatment of GD 10-11 rat conceptuses with sodium formate $(0.5-2 \mathrm{mg} / \mathrm{ml})$ with or without BSO for $24 \mathrm{~h}$ in vitro

\begin{tabular}{|c|c|c|c|c|c|c|}
\hline Treatment & $\begin{array}{c}\% \\
\text { Viability }\end{array}$ & $\begin{array}{c}\% \\
\text { Rotation }\end{array}$ & $\begin{array}{c}\% \\
\text { Closure }\end{array}$ & $\begin{array}{c}\text { Length }(\mathrm{mm}) \\
(\mathrm{n})\end{array}$ & $\begin{array}{l}\text { Somites } \\
\text { (n) }\end{array}$ & $\begin{array}{l}\text { Malformations } \\
\text { (n) }\end{array}$ \\
\hline Control & $\begin{array}{c}100 \\
(26 / 26)\end{array}$ & $\begin{array}{c}100 \\
(26 / 26)\end{array}$ & $\begin{array}{c}92 \\
(22 / 24)\end{array}$ & $\begin{array}{l}3.4 \pm 0.0 \\
\quad(26)\end{array}$ & $\begin{array}{l}25.5 \pm 0.3 \\
\quad(26)\end{array}$ & None \\
\hline $\begin{array}{l}\text { HCOONa } \\
(0.5 \mathrm{mg} / \mathrm{ml})\end{array}$ & $\begin{array}{c}84^{*} \\
(16 / 19)\end{array}$ & $\begin{array}{c}100 \\
(16 / 16)\end{array}$ & $\begin{array}{c}94 \\
(15 / 16)\end{array}$ & $\begin{array}{l}3.4 \pm 0.0 \\
\quad(16)\end{array}$ & $\begin{array}{l}24.7 \pm 0.2 \\
\quad(18)\end{array}$ & Blisters (1) \\
\hline $\begin{array}{l}\mathrm{HCOONa} \\
(2 \mathrm{mg} / \mathrm{ml})\end{array}$ & $\begin{array}{c}40^{*} \\
(6 / 15)\end{array}$ & $\begin{array}{l}100 \\
(6 / 6)\end{array}$ & $\begin{array}{l}100 \\
(6 / 6)\end{array}$ & $\begin{array}{c}3.4 \pm 0.1 \\
(6)\end{array}$ & $\begin{array}{c}20 \pm 1.1^{*} \\
(12)\end{array}$ & Necrotic (2) \\
\hline $\begin{array}{l}\text { BSO } \\
(2 \mathrm{mg} / \mathrm{ml})\end{array}$ & $\begin{array}{c}95 \\
(21 / 22)\end{array}$ & $\begin{array}{c}95 \\
(20 / 21)\end{array}$ & $\begin{array}{c}95 \\
(19 / 20)\end{array}$ & $\begin{array}{c}3.2 \pm 0.1^{*} \\
(20)\end{array}$ & $\begin{array}{l}25.1 \pm 0.3 \\
\quad(20)\end{array}$ & Blisters (1) \\
\hline $\begin{array}{l}\text { BSO } \\
(2 \mathrm{mg} / \mathrm{ml}) \\
\text { HCOONa }(0.5 \mathrm{mg} / \mathrm{ml})\end{array}$ & $\begin{array}{c}71 \\
(12 / 17)\end{array}$ & $\begin{array}{c}83 \\
(10 / 12)\end{array}$ & $\begin{array}{c}100 \\
(12 / 12)\end{array}$ & $\begin{array}{l}3.2 \pm 0.4 \\
\quad(10)\end{array}$ & $\begin{array}{c}22.7 \pm 0.8^{* *} \\
(10)\end{array}$ & $\begin{array}{l}\text { Blisters (1) } \\
\text { Necrotic (5) }\end{array}$ \\
\hline $\begin{array}{l}\text { BSO } \\
(2 \mathrm{mg} / \mathrm{ml}) \\
\text { HCOONa }(2 \mathrm{mg} / \mathrm{ml})\end{array}$ & $\begin{array}{c}27 * * \\
(4 / 15)\end{array}$ & $\begin{array}{c}100 \\
(4 / 4)\end{array}$ & $\begin{array}{l}100 \\
(4 / 4)\end{array}$ & $\begin{array}{c}3.1 \pm 0.4 \\
\text { (4) }\end{array}$ & NA & Necrotic \\
\hline
\end{tabular}

*Significant difference from control $(p<0.05)$.

$* *$ Significant difference from HCOONa only $(p<0.05)$.

At the lower dose $(12 \mathrm{mg} / \mathrm{ml}), \mathrm{H}_{3} \mathrm{COH}$ cotreatment with $\mathrm{BSO}$ did not significantly decrease viability and neuropore closure as compared to conceptuses that received $\mathrm{H}_{3} \mathrm{COH}$ only (Table 1). Embryos treated at this dose did not manifest any blister formation or appear necrotic. At $24 \mathrm{mg} / \mathrm{ml} \mathrm{H}_{3} \mathrm{COH}$ and $\mathrm{BSO}$, embryonic rotation, neuropore and crown-rump length were decreased to a greater extent than conceptuses receiving $\mathrm{H}_{3} \mathrm{COH}$ only. Somite formation was undetectable in $\mathrm{H}_{3} \mathrm{COH}(24 \mathrm{mg} / \mathrm{ml})$ and BSO treated embryos, and blister formation was evident.

Formaldehyde treatment of BSO-pretreated conceptuses caused significant changes to viability and development (Table 2). BSO pretreatment caused a significant decrease in viability, rotation, neuropore closure and somite number from conceptuses receiving $\mathrm{H}_{2} \mathrm{CO}$ only $(3 \mu \mathrm{g} / \mathrm{ml})$. Blisters were observed in some embryos and a high level of necrosis in others. At the higher dose $(6 \mu \mathrm{g} / \mathrm{ml})$, BSO pretreatments cause complete death after the culture period.

Co-treatment with HCOONa $(0.5 \mathrm{mg} / \mathrm{ml})$ and BSO only affected somite number which was significantly different from embryos receiving HCOONa only (Table 3 ). At the higher concentration of HCOONa, treatments exacerbated the lethal effects but had no other effects on surviving embryos' growth parameters and appeared similar to control embryos.

\section{Glutathione and cysteine}

Control embryos contained approximately $14.0( \pm 2.41 ; n=32)$ pmoles $\mathrm{GSH} / \mu \mathrm{g}$ protein and control VYS contained $32.6( \pm 3.86 ; n=$ 30) pmoles $\mathrm{GSH} / \mu \mathrm{g}$ protein (Figure 2). Buthionine sulfoximine treatment alone produced a significant GSH depletion after $24 \mathrm{~h}$ in 


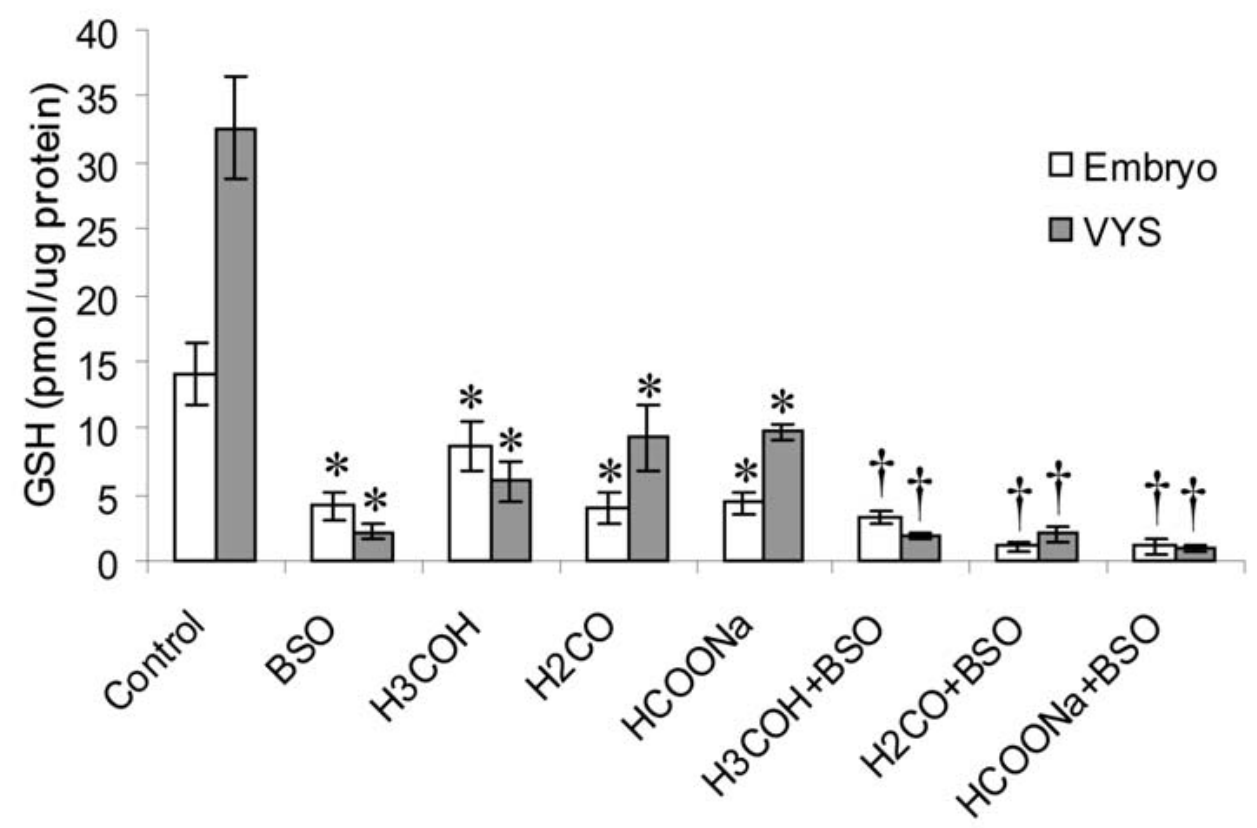

Figure 2. Embryonic glutathione concentrations in GD 11 rat embryos and visceral yolk sacs following buthionine-sulfoximine (BSO), $\mathrm{H}_{3} \mathrm{COH}, \mathrm{CH}_{2} \mathrm{O}, \mathrm{HCOONa}$ and $\mathrm{H}_{3} \mathrm{COH}+\mathrm{BSO}, \mathrm{CH}_{2} \mathrm{O}+\mathrm{BSO}$ and $\mathrm{HCOONa}+\mathrm{BSO}$ in culture for $24 \mathrm{~h}$. Asterisks $(*)$ denote a statistically significant difference $(p<0.05)$ between control. Crosses $(\dagger)$ denote a statistically significant difference $(p<0.05)$ between treatments with and without BSO.

both the embryo and VYS as compared to control conceptuses, measuring $4.1( \pm 1.02)$ and $3.3( \pm 0.53)$ pmoles $\mathrm{GSH} / \mu \mathrm{g}$ protein, respectively (Figure 2 ).

Treatment with $24 \mathrm{mg} / \mathrm{ml} \mathrm{H}_{3} \mathrm{COH}$ resulted in a significant decrease $(p<0.05)$ in embryonic GSH concentrations by $38 \%$ ( 8.7 pmoles $\mathrm{GSH} / \mu \mathrm{g}$ protein $( \pm 1.80 ; n=26))$, but at the same $\mathrm{H}_{3} \mathrm{COH}$ concentrations, GSH was depleted to an even greater extent in the VYS by $83 \%$ (6.0 pmoles $\mathrm{GSH} / \mu \mathrm{g}$ protein $( \pm 1.46 ; n$ = 14)). Treatment with both $24 \mathrm{mg} \mathrm{H}{ }_{3} \mathrm{COH} / \mathrm{ml}$ and BSO caused an even greater GSH depletion that reached significance in both the embryo proper (3.3 pmoles $\mathrm{GSH} / \mu \mathrm{g}$ protein $( \pm 0.54 ; n=24))$ and the VYS (1.9 pmoles $\mathrm{GSH} / \mu \mathrm{g}$ protein $( \pm 0.22 ; n=24))$.

Treatment with $\mathrm{CH}_{2} \mathrm{O}(3 \mu \mathrm{g} / \mathrm{ml})$ caused GSH depletion, measuring $4.0( \pm 1.12 ; n=$ $12)$ and $9.3( \pm 2.41 ; n=12)$ pmoles $\mathrm{GSH} / \mu \mathrm{g}$ protein in the embryo and VYS respectively (Figure 2). Formaldehyde and BSO treatments increased the extent of GSH depletion in both the embryo (1.1 pmoles $\mathrm{GSH} / \mu \mathrm{g}$ protein $( \pm 0.22 ; n=11))$ and VYS $(2.0$ pmoles GSH/ $\mu \mathrm{g}$ protein $( \pm 0.31 ; n=11))$.

Finally, treatment with $\mathrm{HCOONa}$ depleted GSH as well the extent of which was very similar to $\mathrm{CH}_{2} \mathrm{O}$ treated conceptuses, decreasing GSH in the embryo to $4.3( \pm 0.9 ; n=6)$ pmoles $\mathrm{GSH} / \mu \mathrm{g}$ protein and in the VYS to 9.8 $( \pm 0.61 ; n=6) \mathrm{pmoles} \mathrm{GSH} / \mu \mathrm{g}$ protein (Figure 2). Prior treatment with BSO further diminished GSH stores with $\mathrm{HCOONa}$ in both the embryo proper $(1.1( \pm 0.6 ; n=8)$ pmoles $\mathrm{GSH} / \mu \mathrm{g}$ protein $)$ and the VYS $(0.9( \pm 0.22 ; n$ $=8)$ ).

Cysteine was concentrations were also determined. Control VYS contained $5.5( \pm 1.50 ; n$ $=32$ ) pmoles cysteine $/ \mu \mathrm{g}$ protein while control 


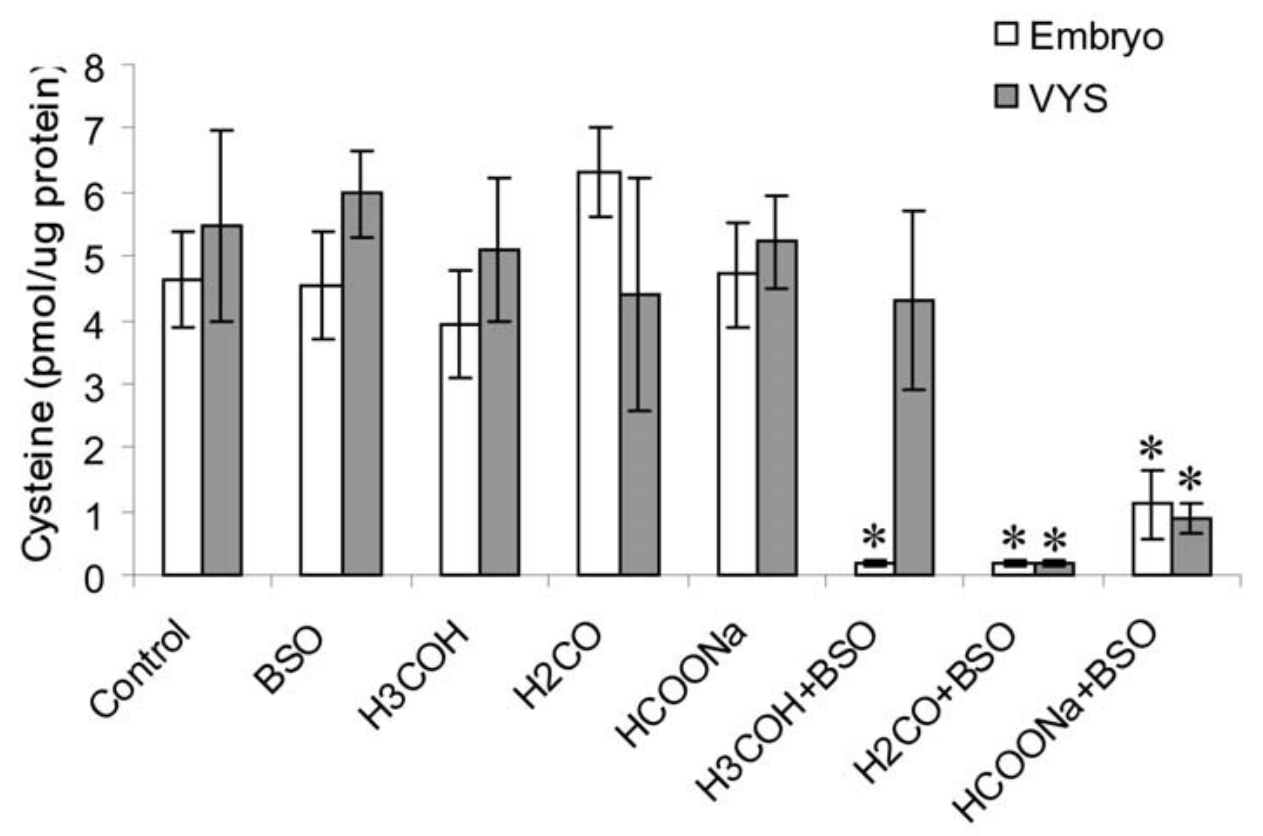

Figure 3. Cysteine concentrations in GD rat embryos and visceral yolk sacs following treatment with $\mathrm{H}_{3} \mathrm{COH}, \mathrm{CH}_{2} \mathrm{O}$ or $\mathrm{HCOONa}$ with or without BSO treatment in culture for $24 \mathrm{~h}$. Asterisks $\left(^{*}\right)$ denote a statistically significant difference $(p<0.05)$ between treatments with and without BSO.

embryos contained $4.6( \pm 0.74 ; n=30)$ pmoles cysteine/ $\mu \mathrm{g}$ protein (Figure 3). Conceptuses that received BSO only did not exhibit a significant decrease in cysteine content.

Methanol treatment $(24 \mathrm{mg} / \mathrm{ml})$ did not have a significant effect on cysteine concentrations in either the embryo proper or VYS (Figure 3). However, with prior BSO treatment, $\mathrm{H}_{3} \mathrm{COH}$ exposure caused a significant cysteine depletion but only in the embryo $(0.2$ $( \pm 0.05 ; n=26))$ pmoles cysteine/ $\mu$ g protein $)$ and not in the VYS $(4.3( \pm 1.40 ; n=14)$ pmoles cysteine/ $\mu$ g protein).

$\mathrm{CH}_{2} \mathrm{O}$ treatment $(3 \mu \mathrm{g} / \mathrm{ml})$ increased cysteine in the embryo proper $(6.3( \pm 0.07 ; n$ $=12$ ) pmoles cysteine $/ \mu$ g protein) but the VYS remained relatively unchanged, measuring 4.4 $( \pm 1.81 ; n=12)$ pmoles cysteine $/ \mu \mathrm{g}$ protein (Figure 3). BSO and $\mathrm{CH}_{2} \mathrm{O}$ co-treatments resulted in a reduction of cysteine pools in both the embryo $(0.2( \pm 0.05 ; n=11)$ pmoles cysteine/ $\mu \mathrm{g}$ protein) and VYS $(0.2( \pm 0.04 ; n$ $=11) \mathrm{pmoles}$ cysteine $/ \mu \mathrm{g}$ protein).

HCOONa exposure $(2 \mathrm{mg} / \mathrm{ml})$ had little effect in the embryo proper or in the VYS (Figure 3). With both $\mathrm{BSO}$ and $\mathrm{HCOONa}$ treatments, cysteine concentrations were significantly reduced in both the embryo (1.1 $( \pm 0.55 ; n=8)$ pmoles cysteine/ $\mu \mathrm{g}$ protein $)$ and VYS $(0.9( \pm 0.24 ; n=8)$ pmoles cysteine/ $\mu \mathrm{g}$ protein).

\section{Discussion}

Previous work to describe $\mathrm{H}_{3} \mathrm{COH}$ and formate toxicity has been performed in rat whole embryo culture and produced similar growth and developmental abnormalities as observed in this study (Andrews et al., 1998). In both rat and mouse embryos, formic acid and HCOONa exposure in vitro resulted in the 
inhibition of anterior and posterior neuropore closure, rotational defects, tail anomalies, enlarged pericardium and delayed heart development (Andrews et al., 1995). In our study, neuropore closure was inhibited by $\mathrm{H}_{3} \mathrm{COH}$ and suggests the initiation of early events that lead to exencephaly, a common defect seen in rodents exposed to $\mathrm{H}_{3} \mathrm{COH}$ via inhalation (Nelson et al., 1985; Bolon et al., 1993). In spite of limitations for whole embryo culture, where neural development cannot be followed to term in vitro to assess permanence of embryonic lesions, the common nature of malformations seen in whole embryo culture and data from in vivo $\mathrm{H}_{3} \mathrm{COH}$ exposures help to validate the extrapolation to in vivo models. Furthermore, our results correlated well with these previous studies which utilized both $\mathrm{H}_{3} \mathrm{COH}$ and formate, but have produced new data suggesting that $\mathrm{CH}_{2} \mathrm{O}$ also contributes to a decrease in conceptus viability. Dysmorphogenesis and other developmental abnormalities are more prevalent in conceptuses treated with $\mathrm{H}_{3} \mathrm{COH}$ and $\mathrm{CH}_{2} \mathrm{O}$ while $\mathrm{HCOONa}$ treatments produced less dysmorphogenic effects and are selectively embryolethal. These data show that $\mathrm{H}_{3} \mathrm{COH}$ and $\mathrm{CH}_{2} \mathrm{O}$, which rely on GSH for detoxication and elimination, are the most dysmorphogenic but that HCOONa, a post-GSH conjugation and elimination metabolite, produces the greatest lethality but no dysmorphogenesis. These results implicate GSH as a critical factor in $\mathrm{H}_{3} \mathrm{COH}$-induced dysmorphogenesis. The lack of dysmorphogenesis seen with HCOONa in the present study does not rule out the possibility of producing malformations but may simply reflect a choice of doses that bracket a very steep dose response.

Depletion of GSH via BSO alone did not effect embryonic development, but subsequent treatment with $\mathrm{H}_{3} \mathrm{COH}$ caused significant developmental abnormalities and decreased viability in a dose-dependent manner. Formaldehyde had a similar effect on development.
Although HCOONa proved to be very toxic, the remaining embryos did not manifest any malformations or altered growth parameters.

While not evaluated specifically in embryos, other studies have shown that $\mathrm{H}_{3} \mathrm{COH}$ exposure in adult rats alters antioxidant enzymes, such as glutathione peroxidase and glutathione disulfide reductase, and antioxidants, such as GSH and ascorbate, in the liver, erythrocytes, brain, and blood serum (Skrzydlewska and Farbiszewski, 1998; Skrzydlewska et al., 1998). Glutathione plays a crucial role in the detoxication of ROS and reactive intermediates. Our study shows that $\mathrm{H}_{3} \mathrm{COH}, \mathrm{CH}_{2} \mathrm{O}$ and HCOONa, at embryotoxic doses, all produce a significant decrease in GSH content in both the embryo and VYS, suggestive that GSH is active in the detoxication of $\mathrm{H}_{3} \mathrm{COH}$ and/or $\mathrm{H}_{3} \mathrm{COH}$-related metabolites.

Another interesting observation is that $\mathrm{H}_{3} \mathrm{COH}$ caused a greater depletion of GSH in the VYS than the embryo, while both $\mathrm{CH}_{2} \mathrm{O}$ and HCOONa showed greater GSH depletion in the embryo proper. Preferential depletion by $\mathrm{H}_{3} \mathrm{COH}$ of VYS GSH suggests that principle site of $\mathrm{H}_{3} \mathrm{COH}$ detoxication is in this extraembryonic organ, which may play a role as a primary line of defense to protect the embryo proper. Unlike human fetal development, the rat embryo is encompassed by the VYS throughout gestation and has approximately $65 \%$ more $\mathrm{GSH}$ ( $\sim 20 \mathrm{pmoles} / \mu \mathrm{g}$ protein $)$ than the embryo proper $(\sim 12 \mathrm{pmoles} / \mu \mathrm{g}$ protein) (Figure 2) (Hansen et al., 1999). Unlike $\mathrm{H}_{3} \mathrm{COH}, \mathrm{CH}_{2} \mathrm{O}$ and $\mathrm{HCOONa}$ both caused a more substantial GSH depletion in the embryo, indicating that both $\mathrm{CH}_{2} \mathrm{O}$ and HCOONa metabolism involving GSH may occur primarily in the embryo rather than the VYS. It should also be considered that a greater apparent GSH depletion occurs in the embryo due to a reduced capacity to supply precursor amino acids for new synthesis or due to an inability to enzymatically reduce oxidized GSH. 
Prior depletion of GSH with BSO followed by subsequent treatments with $\mathrm{H}_{3} \mathrm{COH}, \mathrm{CH}_{2} \mathrm{O}$ or $\mathrm{HCOONa}$ potentiated $\mathrm{GSH}$ depletion as compared to BSO alone and correlated with decreased viability. Growth and developmental parameters were only affected in conceptuses that received $\mathrm{H}_{3} \mathrm{COH}$ and $\mathrm{CH}_{2} \mathrm{O}$ but, interestingly, not HCOONa. These data suggest that HCOONa-mediated effects, namely decreased viability, are most likely not mediated by GSH and could be attributed more to acidosis as previously suggested by other studies (Lanigan, 2001). Decreased GSH in HCOONatreated embryos may be a result of oxidation due to an acidic $\mathrm{pH}$ rather than active detoxication of HCOONa.

Methanol is converted to $\mathrm{CH}_{2} \mathrm{O}$ by $\mathrm{ADH} 1$, located primarily in the liver of adults. However, rat embryonic livers (GD 12-20) have been shown to have very low alcohol dehydrogenase activity (Zorzana and Herrera, 1989) and may not be capable of metabolizing $\mathrm{H}_{3} \mathrm{COH}$, relying on other tissues and/or other methods to metabolize $\mathrm{H}_{3} \mathrm{COH}$. An alternative $\mathrm{H}_{3} \mathrm{COH}$ metabolism pathway is through catalase, producing $\mathrm{CH}_{2} \mathrm{O}$ and hydrogen peroxide (Lanigan, 2001). Previous work in our laboratory showed that rat embryos have $15-25 \%$ less ADH1 and ADH3 specific activity than the VYS during early organogenesis but rat embryo catalase activity is considerably higher than the VYS (Harris et al., 2003), implying that the embryo could better utilize the catalase pathway for $\mathrm{H}_{3} \mathrm{COH}$ conversion to $\mathrm{CH}_{2} \mathrm{O}$. If the catalase detoxication pathway of $\mathrm{H}_{3} \mathrm{COH}$ is primarily utilized by the embryo, it would offer some rationale to GSH depletion through hydrogen peroxide generation, unlike ADH1 metabolism where hydrogen peroxide is not generated. Furthermore, it provides additional evidence that the embryo is a sensitive target for $\mathrm{H}_{3} \mathrm{COH}$ toxicity. An increased generation of hydrogen peroxide as a component of the mechanism of $\mathrm{H}_{3} \mathrm{COH}$ embryotoxicity could also be important in explaining the clear and blood-filled blisters seen in exposed embryos. Generation of reactive oxygen species can produce large regions of $\mathrm{O}_{2}$ depletion and the resulting moderate hypoxia is known to elicit these types of blistering effects in developing embryos.

Formaldehyde exposure had significant effects on viability and development, and these effects were potentiated by GSH depletion with BSO. Formaldehyde affected embryos at a concentration that was considerably lower than $\mathrm{H}_{3} \mathrm{COH}\left(\sim 100-200 \mu \mathrm{mol} / \mathrm{L} \mathrm{CH}_{2} \mathrm{O}\right.$ vs $\sim 375-750 \mathrm{mmol} / \mathrm{L} \mathrm{H}_{3} \mathrm{COH}$ ), suggesting teratogenesis may be a result of $\mathrm{CH}_{2} \mathrm{O}$ rather than $\mathrm{H}_{3} \mathrm{COH}$. Formaldehyde, the main metabolite of $\mathrm{H}_{3} \mathrm{COH}$, relies largely on $\mathrm{GSH}$ for detoxication in rat conceptuses as viability declined and development was disrupted. The conversion of $\mathrm{CH}_{2} \mathrm{O}$ to formate is dependent on $\mathrm{GSH}$, as noted in the introduction (Figure 1), and $\mathrm{GSH}$ depletion by $\mathrm{CH}_{2} \mathrm{O}$ alone may be due to its reactive nature. Decreased concentrations of $\mathrm{GSH}$ would block normal $\mathrm{CH}_{2} \mathrm{O}$ elimination. The inability to form $S$-formylglutathione and the decrease of $\mathrm{CH}_{2} \mathrm{O}$ 's subsequent metabolism to formic acid may result in the accumulation of $\mathrm{CH}_{2} \mathrm{O}$ and cause cell damage and death, eventually contributing to teratogenesis and other undesirable developmental outcomes.

Cysteine is of related interest as it is the ratelimiting precursor for GSH in de novo synthesis. Intriguingly, cysteine concentrations were relatively unaffected with $\mathrm{H}_{3} \mathrm{COH}, \mathrm{CH}_{2} \mathrm{O}$, or HCOONa treatments, indicating that although $\mathrm{H}_{3} \mathrm{COH}, \mathrm{CH}_{2} \mathrm{O}$, or $\mathrm{HCOONa}$ alone are capable of depleting GSH stores, both the embryo and VYS are capable of maintaining sufficient cysteine for de novo GSH synthesis. However, with BSO treatment, GSH synthesis is inhibited. Over time, GSH is lost through normal cellular turnover and may affect other reducing equivalents in the cell, such as cysteine, which, besides GSH, is one of the largest non-protein thiol pools in most cells. In each treatment 
coupled with BSO, the already low embryonic cysteine stores may undergo oxidation to cystine, producing the dramatic pattern of cysteine loss seen in Figure 5. Very little is known about cysteine supply and regulation in the developing conceptus. Our previous studies show that the major source of cysteine to the embryo is through proteolytic degradation of maternal proteins in the VYS. It was speculated that restoration of depleted GSH in the embryo proper was dependent on GSH transported from the VYS in order to supply needed cysteine for new GSH synthesis (Harris, 1993). The intriguing observation that $\mathrm{H}_{3} \mathrm{COH}, \mathrm{H} 2 \mathrm{CO}$, and $\mathrm{HCOONa}$ alone do not affect cysteine levels but result in tissue selective depletions in combination with BSO cannot be explained based on our current knowledge. Cysteine/cystine transport is known to be inducible through insults that elicit oxidative stress and is sensitive to changes in oxygen concentrations (Sato et al., 2001). It is possible that combinations of $\mathrm{BSO}, \mathrm{H}_{3} \mathrm{COH}$ and its metabolites are producing conditions of hypoxia under which the transport of cystine is inhibited and to which the embryo may be selectively sensitive in comparison to the VYS.

Methanol, formaldehyde and formic acid are embryolethal, and methanol and formaldehyde are dysmorphogenic. However, mechanisms by which these compounds are detoxicated in the early organogenesis stage embryo are not completely understood. Glutathione is an important factor in methanol detoxication as it has been implicated in methanol metabolism in the adult liver. Here, we show evidence that glutathione is equally important in the detoxication of methanol and formaldehyde in the developing conceptus. Moreover, these findings suggest that during periods of GSH depletion, formaldehyde most likely accounts for the teratogenic outcomes observed in cultured embryos and formate treatment did not exacerbate malformation but only death.

\section{Acknowledgments}

This work was supported by a grant from the US Environmental Protection Agency (Cr821964) and the National Institutes of Health (NIH ES07062).

\section{References}

Andrews JE, Ebron-McCoy M, Kavlock RJ, Rogers JM. Developmental toxicity of formate and formic acid in whole embryo culture: a comparative study with mouse and rat embryos. Teratology. 1995;51:243-51.

Andrews JE, Ebron-Mccoy M, Schmid JE, Svendsgaard D. Effects of combinations of methanol and formic acid on rat embryos in culture. Teratology. 1998;58:54-61.

Bolon B, Dorman DC, Janszen D, Morgan KT, Welsch F. Phase-specific developmental toxicity in mice following maternal methanol inhalation. Fundam Appl Toxicol. 1993;21:508-16.

Bradford MM. A rapid and sensitive method for the quantitation of microgram quantities of protein utilizing the principle of protein-dye binding. Anal Biochem. 1976;72:248-54.

Fahey RC, Newton GL. Determination of low-molecularweight thiols using monobromobimane fluorescent labeling and high-performance liquid chromatography. Methods Enzymol. 1987;143:85-96.

Fantel AG, Juchau MR, Tracy JW, Burroughs CJ, Person RE. Studies of mechanisms of niridazole-elicited embryotoxicity: evidence against a major role for covalent binding. Teratology. 1989;39:63-74.

Farbiszewski R, Witek A, Skrzydlewska E. N-acetylcysteine or trolox derivative mitigate the toxic effects of methanol on the antioxidant system of rat brain. Toxicology. 2000;156: $47-55$.

Food and Drug Administration (FDA). 1984. Frequency of use of cosmetic ingredients. FDA Database. Washington, DC: FDA.

Hansen JM, Carney EW, Harris C. Differential alteration by thalidomide of the glutathione content of rat vs. rabbit conceptuses in vitro. Repro Toxicol. 1999;13:547-54.

Harris C, Namkung MJ, Juchau MR. Regulation of intracellular glutathione in rat embryos and visceral yolk sacs and its effect on 2-nitrosofluorene-induced malformations in the whole embryo culture system. Toxicol Appl Pharmacol. 1987;88:141-52.

Harris C, Juchau MR, Mirkes PE. Role of glutathione and hsp 70 in the acquisition of thermotolerance in postimplantation rat embryos. Teratology. 1991;43:229-39.

Harris, C. Glutathione biosynthesis in the postimplantation rat conceptus in vitro. Toxicol Appl Pharmacol. 1993;120: 24756.

Harris C, Wang S-W, Lauchu JJ, Hansen JM. Methanol metabolism and embryotoxicity in rat and mouse conceptuses: Comparisons of alcohol dehydrogenase (ADH1), 
formaldehyde dehydrogenase (ADH3), and catalase. Repro Toxicol. 2003;17:349-57.

Infurna R, Weiss B. Neonatal behavioral toxicity in rats following prenatal exposure to methanol. Teratology. 1986; 33:259-65.

Kavet R, Nauss KM. The toxicity of inhaled methanol vapors. Crit Rev Toxicol. 1990;21:21-50.

Lanigan S. Final report on the safety assessment of methyl alcohol. Int J Toxicol. 2001;20(Suppl. 1):57-85.

Nelson BK, Brightwell WS, MacKenzie DR, et al. Teratological assessment of methanol and ethanol at high inhalation levels in rats. Fundam. Appl Toxicol. 1985;5:727-36.

Sato H, Kuriyama-Matsumura K, Hashimoto T, et al. Effect of oxygen on induction of the cysteine transporter by bacterial lipopolysaccharide in mouse peritoneal macrophages. J Biol Chem. 2001;276:10407-12.

Skrzydlewska E, Farbiszewski R. Lipid peroxidation and antioxidant status in the liver, erythrocytes, and serum of rats after methanol intoxication. J Toxicol Environ Health. 1998;53:637-49.

Skrzydlewska E, Witek A, Farbiszewski R. The comparison of the antioxidant defense potential of brain to liver of rats after methanol ingestion. Comp Biochem Physiol C Pharmacol Toxicol Endocrinol. 1998;120:289-94.

Von Burg R. Methanol. J Appl Toxicol. 1994;14:309-13.

Ward KW, Pollack GM. Maternal-fetal toxicokinetics of methanol. Toxicologist. 1995; 15:186.

Ward KW, Pollack GM. Comparative toxicokinetics of methanol in pregnant and nonpregnant rodents. Drug Metab Dispos. 1996;24:1062-70.

Youssef AF, Baggs RB, Weiss B, Miller RK. Teratogenicity of methanol following a single oral dose in Long-Evans rats. Reprod Toxicol. 1997;11:503-10.

Zorzano A, Herrera E. Decreased in vivo rate of ethanol metabolism in the suckling rat. Alcohol Clin Exp Res. 1989;13:527-32.

Address for correspondence: Craig Harris, Toxicology Program, Department of Environmental Health Sciences, The University of Michigan, 1420 Washington Heights, Ann Arbor, Michigan, 48109-2029, USA.

E-mail: charris@umich.edu 